\title{
ENFOQUE DIAGNÓSTICO DE LA HEMATURIA EN PEDIATRÍA
}

Helen Preciado MD*, Natalia Cáceres MD**, Carlos Beltrán MD**, Héctor Ortiz MD**

\section{Resumen}

L a hematuria es un hallazgo clínico observado por el paciente o un familiar cuando es macroscópica o bien incidental en un parcial de orina rutinario. L a prevalencia es baja pero el enfoque diagnóstico al cual está enfrentado el clínico es amplio. L a hematuria indica enfermedad, lesión o malformación del tracto urinario, por ello el médico general y el pediatra deben estar en capacidad de determinar a tiempo algunas de las causas con pronóstico grave o diagnóstico complejo que requieren la remisión al nefrólogo pediatra. Se hace énfasis en diferenciar si es o no de origen glomerular, adoptando un algoritmo de enfoque diagnóstico que orienta al clínico. Se realizó una búsqueda limitada de 2001-2014 en las principales bases de datos en inglés y español encontrando revisiones de la literatura, consensos de expertos y guías de práctica clínica basadas en la opinión de pediatras, nefrólogos y urólogos pediatras, así como de radiólogos que ofrecen una aproximación real a la imagenología. E stá dirigida a personal de la salud, médicos generales y pediatras quienes enfrentan a diario esta condición clínica.

Palabras clave: hematuria, microscópica, macroscópica, glomerular, no glomerular.

\section{DIAGNOSTIC APPROACH TO A CHILD WITH HEMATURIA}

\begin{abstract}
Hematuria is a clinical finding observed by the patient or a relative if it is macroscopic, or is an incidental finding in a routine urinalysis. Prevalence rate is low but the range of differential diagnoses faced by the physician is broad. Hematuria indicates disease, lesion or malformation of the urinary tract, thus the general practitioner and the pediatrician must be capable to determine the etiologies that have a poor prognosis or are difficult to diagnose which require prompt referral to a pediatric nephrologist. We emphasize on the development of a diagnostic algorithm to help the physician distinguish glomerular from non-glomerular hematuria. A limited search from 2001-2014 was conducted in the major data bases in E nglish and Spanish finding literature reviews, expert consensus and clinical practice guidelines based on the opinion of pediatricians, and pediatric nephrologists and urologists, as well as radiologists who offer a real approach to imaging. It is oriented toward health care providers, general practitioners and pediatricians who face this clinical condition in their daily practice.
\end{abstract}

Key words: hematuria, microscopic, macroscopic, glomerular, non-glomerular

Fecha recibido: junio 4 de 2014 - Fecha aceptado: diciembre 5 de 2014

* Pediatra, Instructor Asistente, Fundación Universitaria de Ciencias de la Salud. Bogotá DC, Colombia.
** Residente III de Pediatría. Fundación Universitaria de Ciencias de la Salud. Bogotá DC, Colombia. 


\section{Introducción}

La hematuria indica enfermedad, lesión o malformación del tracto urinario. Es el hallazgo clínico más frecuente dentro de la patología genitourinaria después de la infección. La incidencia de la variante microscópica entre los niños de edad escolar oscila entre 0,4 y $2 \%$. Pese a ello, en algunos casos es una manifestación de enfermedad grave., ${ }^{1,2}$

Hay una larga lista de causas de hematuria, la mayoría de las cuales son benignas, en especial cuando es aislada, microscópica y asintomática. El dilema que enfrenta el clínico es identificar la causada por una enfermedad significativa. El hecho de realizar un adecuado enfoque diagnóstico permite al clínico orientar la sintomatología y así tener clara la situación que amerita la remisión oportuna al nefrólogo pediatra.

\section{Justificación}

La hematuria es un hallazgo frecuente en los niños. Como se ilustra en dos grandes estudios poblacionales, de 3 a $4 \%$ en edad escolar entre 6 y 15 años tenía una tira reactiva positiva para sangre en una sola muestra de orina. ${ }^{2}$

Varios estudios poblacionales de niños en edad escolar han demostrado que la tasa de prevalencia de la microscópica detectada en una sola muestra de orina es de 3 a $4 \%$, lo que corresponde a $1 \%$ o menos con dos o más muestras positivas, de los cuales sólo un tercio tiene hematuria persistente, definida como una repetición de la prueba positiva después de seis meses. La combinación con proteinuria es menos común, cuya prevalencia es menor del $0,7 \%$ en niños en edad escolar en una sola muestra de orina. ${ }^{2,3}$

La detección por parte de la familia de sangre en la orina (macroscópica) es motivo de inquietud y angustia que conlleva consulta inmediata y en diversas ocasiones a acudir al servicio de urgencias. Sin embargo, no es la forma de presentación usual. Con frecuencia se trata de niños en los que no existen alteraciones detectables a simple vista en la orina y que el examen con una tira reactiva (multistix) evidencia la presencia de sangre (microscópica). Lo anterior es una diferencia básica porque gran parte de los niños que consultan por hematuria macroscópica requerirán ingreso, mientras un buen porcentaje de los que la presentan microscópica serán tratados en forma ambulatoria. ${ }^{4}$

La clave del manejo en urgencias radica en diferenciar la hematuria glomerular de la no glomerular y determinar la etiología, pronóstico y posibilidades terapéuticas. Esto exige al pediatra a realizar una correcta valoración inicial, diferenciando aquellas situaciones que por su carácter benigno no precisan de exploraciones agresivas, de otras que exigirán un estudio más completo y su derivación al nefrólogo pediatra.

\section{Materiales y métodos}

La guía de práctica clínica del enfoque diagnóstico de la hematuria en pediatría se basa en la adopción y adaptación de guías en inglés a nivel internacional y algunas en español que permiten el enfoque adecuado de ésta sintomatología que aunque poco común, exige una adecuada orientación clínica. Se definió la estrategia $P I C O$ para realizar la búsqueda de la literatura. Población: pacientes que acuden a urgencias o consulta externa con cuadro de hematuria. Intervención: realizar diagnóstico imagenológico. Comparador: llegar a un diagnóstico clínico. Desenlaces: tratamiento oportuno y/o remisión al nefrólogo pediatra. Para ello se realizó una búsqueda basada en términos definidos en descriptores en ciencias de la salud (DECS) y Medical subject headings (MESH). La búsqueda se limita a 2001-2014 en bases de datos en inglés que además puedan ofrecer guías de práctica clínica a nivel mundial como Dynamed y otros artículos relacionados en PUBMED y OVID. Se utilizó la revisión de UptoDate, así como guías y datos en español en la base de datos LILACS y SCIELO. Dentro de los consensos y guías de relevancia clínica para el grupo se revisaron 40 documentos, de ellos cinco cumplían los criterios de elegibilidad, dentro de los cuales se encuentran el consenso de evaluación de la hematuria del grupo de nefrología pediátrica de la India de 2006, así como también las guías de la sociedad japonesa de nefrología pediátrica y urología 2008. Para la adopción de recomendaciones imagenológicas el grupo investigador 
se basó en las del Colegio Americano de Radiología año 2012 en el estudio de la hematuria en el paciente pediátrico. Para su recopilación se usó el método Delphi, basado en la opinión de expertos, que la clasifica como por lo regular no apropiada con una valoración de 1 a 3, puede ser apropiada de 4 a 6 y usualmente apropiada de 7 a 9.

Población: la guía está dirigida al profesional de la salud en general y a médicos y pediatras en particular que atienden al lactante menor o mayor, preescolar, escolar y adolescente que acuden a urgencias o consulta externa por hallazgo de hematuria ya sea micro o macroscópica.

\section{Aspectos clínicos centrales}

Los pediatras se enfrentan con frecuencia a la presencia de hematuria. La incidencia en niños se estima en $0,13 \%$. En más de la mitad de los casos $(56 \%)$ se debe a una causa fácil de identificar. ${ }^{5} \mathrm{La}$ microscópica asintomática es diez veces más frecuente que la macroscópica. Muchos casos de la primera son transitorios y ante repetidas evaluaciones la prevalencia disminuye a menos del 0,5\%. Si hay hematuria y proteinuria puede coexistir una enfermedad renal significativa. ${ }^{1,2}$

Los niños consultan por hematuria macroscópica, síntomas urinarios, como hallazgo incidental de hematuria microscópica o bien es un descubrimiento inadvertido durante un análisis rutinario de orina. La forma macroscópica la refieren por el color tipo " $\mathrm{Co}$ ca-Cola". Una pequeña cantidad de sangre $(1 \mathrm{ml}$ en $1.000 \mathrm{ml}$ de orina) es suficiente para hacer que luzca de color rojo. La reacción positiva en el análisis exige examen microscópico para confirmar la presencia de glóbulos rojos.

La tira reactiva utiliza la actividad pseudoperoxidasa de la hemoglobina (o mioglobina) para catalizar una reacción entre peróxido de hidrógeno y el cromógeno tetrametilbenzidina, para producir una oxidación del cromógeno que tiene un color verde-azul. Es importante sumergir brevemente la tira en la orina y leerla en el tiempo recomendado (por lo general un minuto). ${ }^{6}$
Tiene una sensibilidad de $100 \%$ y una especificidad de $99 \%{ }^{7}$

La confirmación de la hematuria microscópica por el examen de la orina requiere la identificación de más de cinco glóbulos rojos. ${ }^{2,8}$ Se hace mediante la centrifugación de $10 \mathrm{ml}$ de una muestra de orina fresca a 2.000 rpm durante 5 minutos decantando el sobrenadante para volver a suspender el sedimento en los 0,5 $\mathrm{ml}$ restantes. El sedimento es examinado por microscopía de gran aumento, contando los eritrocitos en veinte campos y el promedio se reporta. En ausencia de hematuria macroscópica, el hallazgo persistente de hematuria en al menos dos de los tres análisis de orina realizados durante dos a tres semanas garantiza mayor evaluación. ${ }^{9,10}$

El resultado positivo de la tira reactiva y la ausencia de glóbulos rojos y cilindros hemáticos en la orina sugieren hemoglobinuria o mioglobinuria. Una prueba simple consiste en centrifugar una muestra de orina fresca y evaluar el color del sobrenadante; en hemoglobinuria este será de color rosa claro con depósitos mínimos o inexistentes. La ausencia de hemoglobina, glóbulos rojos o mioglobina debe motivar la búsqueda de otras causas de orina de color rojo ya que varias sustancias pueden alterar la coloración de la orina. ${ }^{11}$

\section{Causas de hematuria}

Puede ser de origen glomerular o no. La historia detallada, el examen físico completo y los exámenes de laboratorio pertinentes son indispensables para su evaluación. En casos confirmados el siguiente paso es la localización de su origen. La historia de edema periorbitario matutino, oliguria, orina de color oscuro y la presencia de edema y/o hipertensión en el examen, sugieren una causa glomerular. Además, esta es indolora y con frecuencia muestra glóbulos rojos y células dismórficas. ${ }^{12-14}$

La identificación de hematíes dismórficos se hace en microscopía de luz previa tinción de Wright o en microscopía de contraste. Si al examinar cien glóbulos rojos más del $20 \%$ son dismórficos se piensa en origen glomerular, con una sensibilidad de $96 \%$ y especificidad de $93 \% .^{12,15,16}$ Es un marcador bastan- 
te específico para glomerulonefritis. ${ }^{17}$ La proteinuria puede estar presente independiente de la causa del sangrado, pero por lo general no excede $2+(100 \mathrm{mg} /$ dl). La combinación de hematuria y proteinuria ( $>100$ $\mathrm{mg} / \mathrm{dl}$ ) indica una enfermedad renal significativa de origen glomerular. La cantidad de proteína excretada se evalúa bien por la determinación de la relación de proteína/creatinina en una muestra de orina aislada o por la cuantificación en muestra de 24 horas.

\section{Hematuria glomerular}

La causa más común de hematuria glomerular macroscópica en los niños es la glomerulonefritis postinfecciosa. Al interrogatorio se debe indagar sobre historia de cefalea, vómito, visión borrosa, alteración del sensorio o convulsiones. La ocurrencia de faringitis o infección cutánea dentro de las últimas dos a cuatro semanas sugiere glomerulonefritis posestreptocócica. La historia de artralgias, erupciones en la piel y fiebre prolongada en adolescentes hace pensar en un trastorno autoinmune. La palidez (anemia) no se explica solo por hematuria por lo cual deben estudiarse otras condiciones como lupus eritematoso sistémico. Las erupciones cutáneas y la artritis pueden ocurrir tanto en la púrpura de Henoch Schönlein como en lupus eritematoso sistémico. Por tanto se deben realizar parcial de orina, cultivo, hemograma completo, anticuerpos antiestreptolisina $\mathrm{O}$, nitrógeno proteico, creatinina, electrolitos y siempre que sea posible, niveles de complemento $\left(\mathrm{C}_{3}\right)$. Si estas investigaciones son indicativas de una reciente infección por estreptococo se tratará de una glomerulonefritis, pero cuando son negativas y no hay resolución de síntomas, el diagnóstico será presuntivo de glomerolonefritis posinfecciosa. Varias bacterias y virus han sido implicados como etiología. Cuando cursa con bajos niveles de $\mathrm{C}_{3}$ se hará nueva determinación después de doce semanas para asegurar que han vuelto a la normalidad. Si las características de la nefritis persisten o el diagnóstico no está claro, el paciente debe remitirse al nefrólogo pediátrico. ${ }^{14}$

Aunque no siempre es necesaria la biopsia en los niños con nefritis aguda, se recomienda cuando aparece insuficiencia renal grave o progresiva, proteinuria en rango nefrótico, hematuria macroscópica o hipertensión persistente por más de cuatro semanas. El síndrome nefrítico agudo asociado con características sistémicas e hipocomplementemia persistente por más de doce semanas también puede requerir biopsia. ${ }^{18}$ Muchos pacientes con glomerolonefritis posinfecciosa pueden mostrar hematuria microscópica persistente durante dos años.

La glomerulonefritis rápidamente progresiva se presenta con síntomas y signos similares a la posinfecciosa, pero los estudios de laboratorio muestran insuficiencia renal progresiva. En estos casos se requiere la atención urgente de un nefrólogo pediátrico, ya que un diagnóstico precoz mediante biopsia y tratamiento inmunosupresor agresivo puede prevenir la progresión de la enfermedad renal. ${ }^{14}$ La hematuria microscópica también se puede observar en $20 \%$ de los niños con síndrome nefrótico por enfermedad renal de cambios mínimos y en ocasiones ser recurrente.

Al resolver el cuadro clínico puede ocurrir hematuria microscópica entre los episodios de hematuria macroscópica, caso en el cual hay que sospechar nefropatía IgA o síndrome de Alport que deben aclararse mediante la demostración de depósitos mesangiales de IgA. ${ }^{19}$ Otras condiciones que cursan con hematuria y requieren biopsia renal para el diagnóstico son glomerulonefritis crescéntica, nefritis lúpica, hematuria benigna familiar, síndrome de Alport, nefritis intersticial, glomerulonefritis membranoproliferativa o focal, glomeruloesclerosis segmentaria y enfermedad de Goodpasture. La hematuria familiar o hereditaria puede ser benigna no progresiva o progresiva (síndrome de Alport). Este último se caracteriza por hematuria microscópica recurrente o persistente, alta frecuencia de insuficiencia renal progresiva y pérdida de la audición neurosensorial con defectos oftalmológicos (catarata). El diagnóstico se confirma en la biopsia renal. La historia familiar debe incluir preguntas acerca de la hematuria, insuficiencia renal, pérdida de la audición o defectos visuales, nefrolitiasis y diátesis hemorrágica en familiares de primer grado. ${ }^{19}$ 


\section{La hematuria no glomerular}

Las causas comunes de hematuria no glomerular son la infección del tracto urinario y nefrolitiasis. La cistitis hematúrica sugiere infección del tracto urinario superior. Tanto la infección como la litiasis pueden causar dolor (en el flanco o abdominal). Otros síntomas son disuria, frecuencia, urgencia y posibilidad de fiebre. Cuando la hematuria ocurre en la iniciación o la terminación de la micción, su origen es uretra o vejiga. El crecimiento bacteriano en el cultivo indica infección, requiere tratamiento antibiótico y posterior evaluación radiológica por la posibilidad de obstrucción, reflujo vesicoureteral, enfermedad quística y otras anomalías. ${ }^{20}$

Los antecedentes familiares de cálculos renales o cólicos con hematuria sugieren su presencia. La exploración para cálculos incluye radiografía simple y ecografía de riñones, uréteres y vejiga. Cuando la sospecha clínica es alta y no se visualizan con los estudios mencionados, la tomografía computarizada helicoidal es la modalidad de imagen de elección. La muestra de orina debe enviarse para determinar la relación calcio/ creatinina. Si el resultado es anormal debe recolectarse la orina de 24 horas para confirmar el diagnóstico de hipercalciuria, excepto en niños muy pequeños por la dificultad de hacerlo. La hipercalciuria se sospecha cuando la relación calcio/creatinina de orina al azar es mayor de la esperada para la edad ( $<7$ meses: 0,8 ; 7 a 18 meses: 0,6; 19 meses a 6 años: 0,4; > 6 años: $0,2)$ y se confirma si en la recolección de 24 horas el calcio es alto ( $>4 \mathrm{mg} / \mathrm{k}$ ). La hipercalciuria puede causar hematuria recurrente macro o microscópica como resultado de la irritación del epitelio de la vejiga por microcálculos. Los niños con hipercalciuria idiopática tienen aumentada la excreción urinaria de calcio y cursan con hematuria, incluso en ausencia de cálculos en los estudios de imagen. Algunos de estos niños no desarrollan urolitiasis. La hipercalciuria también puede ocurrir en estados como hiperparatiroidismo, inmovilización prolongada e intoxicación por vitamina D. El ácido úrico urinario se investiga si no hay otra causa de hematuria. Los trastornos hemorrágicos y los defectos de la coagulación pueden presentarse también como hematuria micro o macroscópica, como sucede en hemofilia clásica y en algunos casos de enfermedad de von Willebrand puede ser la primera manifestación. ${ }^{20}$ La presencia de masa abdominal sugerirá un tumor, hidronefrosis o enfermedad renal poliquística. La neoplasia maligna más común en la infancia es el tumor Wilms.

\section{Causas raras}

Hay causas inusuales de hematuria transitoria como trauma, ejercicio extenuante, infecciones, medicamentos, toxinas o la menstruación. Está relacionada con el trastorno de base y desaparece una vez que se resuelve la enfermedad primaria. Ocurre en 3 a $7 \%$ en casos de enfermedad de células falciformes debido a necrosis papilar renal. El diagnóstico se sospecha debido a las características clínicas y se confirma con la electroforesis de hemoglobina. En las mujeres jóvenes con hematuria recurrente, es importante preguntar acerca de un historial de abuso o inserción de un cuerpo extraño vaginal.

\section{Desenlaces previstos}

El estudio adecuado y correcto de la historia elevará la detección de trastornos relacionados, aumentará los diagnósticos tempranos con intervenciones basadas en la evidencia y será mayor al conocimiento del personal de salud sobre el impacto que tiene la remisión temprana a nefrología pediátrica.

\section{Preguntas y recomendaciones}

\section{¿Qué puede causar orina roja sin hematuria?}

Recomendación: los expertos así como el grupo desarrollador de la guía sugieren como punto de buena práctica clínica la identificación de causas de orina oscura sin hematuria las siguientes según la coloración:

Orina roja: células rojas, hemoglobina libre, mioglobina, uratos, medicamentos como cloroquina, remolacha $\mathrm{o}$ alimentos con tinte rojo y porfirinas. 
Orina amarilla o naranja: orina concentrada y rifampicina.

Orina negra o café: pigmentos biliares, metahemoglobinemia y ácido homogentísico.

\section{¿Cuáles son las posibles causas de hematuria?}

Recomendación: el enfoque depende del origen ya sea glomerular o no glomerular y se hará una evaluación clínica para orientar y hacer uso racional de las ayudas diagnósticas. Para ello se toma el algoritmo propuesto por el grupo de nefrología pediátrica de la India y su respectiva asociación pediátrica (Figura $\mathbf{1}){ }^{21}$

\section{¿Qué pruebas de laboratorio son útiles para evaluar la hematuria?}

Recomendación: el análisis de los consensos de expertos en las guías de manejo internacional y latinoamericano permiten concluir que las ayudas de laboratorio que deben realizarse en primera instancia son: uroanálisis, nitrógeno ureico, creatinina sérica, electrolitos en suero, antiestreptolisina $\mathrm{O}$, hemograma, complemento $\mathrm{C} 3$, relación proteinuria/creatinuria en muestra aislada, proteína total, albúmina, colesterol, ANAS, anti DNA, ANCAS, cultivo de orina y relación calciuria/creatinuria en muestra aislada o en orina de 24 horas.

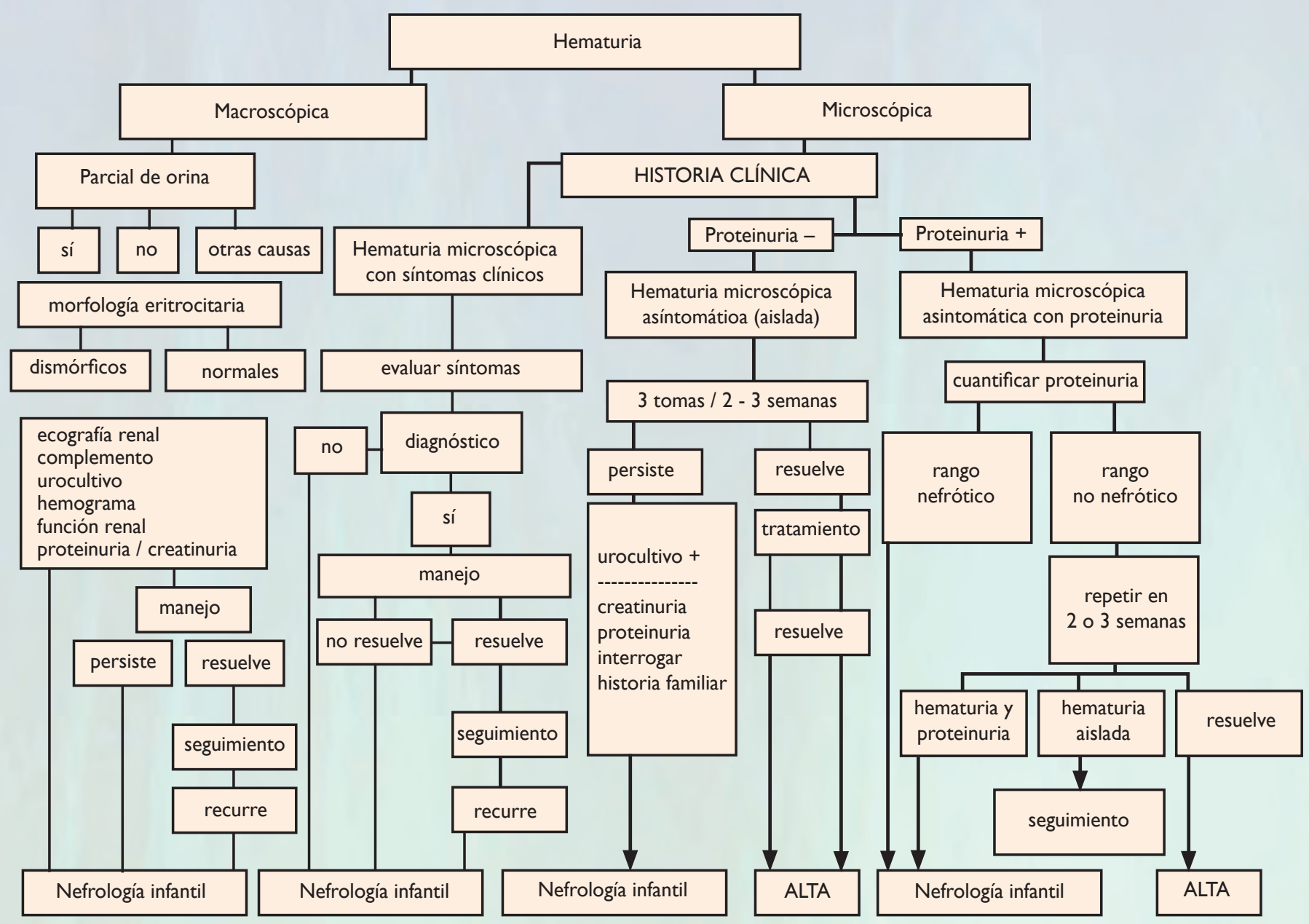

Figura I. Algoritmo de la evaluación práctica de la hematuria. Modificado de Vara J HE, García J. Diagnóstico de la hematuria. Protocolos Diagnósticos y terapéuticos de la AEP. 2008;1:169-81.21 
El Colegio Americano de Radiología en 2012 publicó las recomendaciones sobre el uso apropiado de la imagenología en el estudio de la hematuria en el paciente pediátrico. Para su recopilación se usó el método Delphi el cual se basa en la opinión de expertos. Se clasifica la recomendación así: por lo regular no apropiada con una valoración de 1 a 3 , puede ser apropiada de 4 a 6 y usualmente apropiada de 7 a 9 . Se presentan las recomendaciones de acuerdo con las diferentes variantes de presentación de la hematuria. ${ }^{22}$

\section{Hematuria aislada}

- Ecografía de riñón y vejiga: (7) usualmente apropiada.

- Cistouretrografía miccional: (3) por lo regular no apropiada, puede utilizarse si hay anormalidad en la ecografía.

- Tomografía computarizada de abdomen y pelvis sin contraste: (3) por lo regular no apropiada.

- Tomografía computarizada de abdomen y pelvis con contraste: (3) por lo regular no apropiada.

- Resonancia magnética de abdomen y pelvis con y sin contraste: (3) por lo regular no apropiada.

- Radiografía de abdomen y pelvis: (2) por lo regular no apropiada.

- Arteriografía renal: (2) por lo regular no apropiada.

- Urografía excretora: (2) por lo regular no apropiada.

\section{Hematuria no traumática dolorosa (no glomerular)}

- Tomografía computarizada de abdomen y pelvis sin contraste: (8) usualmente apropiada.

- Ecografía renal y de vías urinarias: (8) usualmente apropiada.

Exámenes alternativos: si la ecografía es negativa, la tomografía puede estar clínicamente indicada y en algunos casos puede detectar otras causas de hematuria dolorosa como obstrucción o tumor renal.
- Radiografía de abdomen y pelvis: (6) puede ser apropiada.

- Resonancia, arteriografía renal, urografía excretora, cistouretrografía: (2) por lo regular no apropiada.

\section{Hematuria macroscópica traumática}

- Tomografía computarizada de abdomen y pelvis con contraste: (9) usualmente apropiada.

- Uretrografía retrograda: (6) puede ser apropiada si la sangre está presente en el meato uretral o si hay fractura pélvica o pelviureteral.

- TAC de pelvis o vejiga sin contraste: (5) puede ser apropiada.

- Arteriografía renal: (3) puede ser apropiada solo para intervención terapéutica.

- Ecografía renal y de vías urinarias: (3) por lo regular no apropiada.

- Radiografía de abdomen y pelvis, resonancia, TAC sin contrastes, urografía excretora: (2) por lo regular no apropiada.

\section{Hematuria microscópica traumática}

- Tomografía computarizada de abdomen y pelvis con contraste: (7) usualmente apropiada.

- Ecografía renal y de vías urinarias: (4) puede ser apropiada.

- Uretrografía retrograda: (6) puede ser apropiada si la sangre está presente en el meato uretral o si hay fractura pélvica o pelviureteral.

- TAC de pelvis o vejiga sin contraste: (5) puede ser apropiada.

- Arteriografía renal, radiografía de abdomen y pelvis, cistouretrografía, urografía excretora, resonancia y TAC con o sin contraste: (2) por lo regular no apropiada. 
Una presentación poco frecuente de hematuria cíclica en los niños adolescentes se ve en la menarquia de las mujeres con hiperplasia adrenal congénita y virilización, que han sido planteadas como varones. La remisión a un cirujano pediatra o urólogo se requiere cuando hay una anomalía estructural urogenital o sospecha de un cálculo obstructivo o tumor. La cistoscopia rara vez es necesaria en los niños como estudio de rutina ante hematuria no glomerular. ${ }^{20}$

\section{Referencias}

1. Vehaskari VM, Rapola J, Koskimies O, Savilahti E, Vilska J, Hallman N. Microscopic hematuria in school children: epidemiology and clinicopathologic evaluation. J Pediatr. 1979; 95(5 Pt 1):676-84.

2. Dodge WF, West EF, Smith EH, Bruce H, 3rd. Proteinuria and hematuria in schoolchildren: epidemiology and early natural history. J Pediatr. 1976; 88(2): $327-47$.

3. Iitaka K, Igarashi S, Sakai T. Hypocomplementaemia and membranoproliferative glomerulonephritis in school urinary screening in Japan. Pediatr Nephrol. 1994;8(4):420-2.

4. Vara Martin J, Hidalgo-Barquero del Rosal E, García Blanco JM. Diagnóstico de la hematuria. Protocolos Diagnósticos y terapéuticos de la AEP. 2008;1:169-81.

5. Ingelfinger JR, Davis AE, Grupe WE. Frequency and etiology of gross hematuria in a general pediatric setting. Pediatrics. 1977;59(4):557-61.

6. Kher K, Schnaper HW, Makker SP. Clinical pediatric nephrology. $2^{\text {nd }}$ ed. London: Taylor \& Francis; 2006.

7. Moore GP, Robinson M. Do urine dipsticks reliably predict microhematuria? The bloody truth!. Ann Emerg Med. 1988;17(3):257-60.
8. Fassett RG, Horgan BA, Mathew TH. Detection of glomerular bleeding by phase-contrast microscopy. Lancet. 1982;1(8287):1432-4.

9. Feld LG, Waz WR, Perez LM, Joseph DB. Hematuria. An integrated medical and surgical approach. Pediatr Clin North Am. 1997; 44(5): 1191-210.

10. Diven SC, Travis LB. A practical primary care approach to hematuria in children. Pediatr Nephrol. 2000;14(1):65-72.

11. Kalia A, Travis L. Hematuria, leukocyturia and cylinduria. In: Edelmann CM, editor. Pediatric kidney disease. 2nd ed. Boston: Little Brown; 1992. p. 553-63.

12. Vijayakumar M, Nammalwar BR. Diagnostic approach to a child with hematuria. Indian Pediatr. 1998; 35(6):525-32.

13. Meyers KE. Evaluation of hematuria in children. Urol Clin North Am. 2004;31(3):559-73, x.

14. Kincaid-Smith P, Fairley K. The investigation of hematuria. Semin Nephrol. 2005; 25(3):127-35.

15. Stapleton FB. Morphology of urinary red blood cells: a simple guide in localizing the site of hematuria. Pediatr Clin North Am. 1987;34(3):561-9.

16. Mehta K, Tirthani D, Ali U. Urinary red cell morphology to detect site of hematuria. Indian Pediatr. 1994;31(9):1039-45.

17. Cilento BG, Jr., Stock JA, Kaplan GW. Hematuria in children. A practical approach. Urol Clin North Am. 1995;22(1):43-55.

18. Vijayakumar M. Acute and crescentic glomerulonephritis. Indian J Pediatr. 2002;69(12):1071-5

19. Kashtan CE. Familial hematuria. Pediatr Nephrol. 2009;24(10):1951-8.

20. Stapleton FB. Hematuria associated with hypercalciuria and hyperuricosuria: a practical approach. Pediatr Nephrol. 1994;8(6):756-61.

21. Phadke KD, Vijayakumar M, Sharma J, Iyengar A, Indian Pediatric Nephrology Group. Consensus statement on evaluation of hematuria. Indian Pediatr. 2006;43(11):965-73.

22. Milla SS, Coley BD, Karmazyn B, Dempsey-Robertson ME, Dillman JR, Dory $\mathrm{CE}$, et al. ACR Appropriateness Criteria(R) limping child--ages 0 to 5 years. J Am Coll Radiol. 2012;9(8):545-53. 